\title{
COMPLEXITY OF TERMS, COMPOSITION, AND HYPERSUBSTITUTION
}

\author{
KLAUS DENECKE and SHELLY L. WISMATH
}

Received 25 February 2002

\begin{abstract}
We consider four useful measures of the complexity of a term: the maximum depth (usually called the depth), the minimum depth, the variable count, and the operation count. For each of these, we produce a formula for the complexity of the composition $S_{m}^{n}\left(s, t_{1}, \ldots, t_{n}\right)$ in terms of the complexity of the inputs $s, t_{1}, \ldots, t_{n}$. As a corollary, we also obtain formulas for the complexity of $\hat{\sigma}[t]$ in terms of the complexity of $t$ when $t$ is a compound term and $\sigma$ is a hypersubstitution. We then apply these formulas to the theory of $M$-solid varieties, examining the $k$-normalization chains of a variety with respect to the four complexity measures.

2000 Mathematics Subject Classification: 08A62, 08B15, 08A70.
\end{abstract}

1. Introduction. Let $\tau=\left(n_{i}\right)_{i \in I}$ be a type of algebras with operation symbols $f_{i}$ of arity $n_{i}$ indexed by some set $I$. Let $X=\left\{x_{1}, x_{2}, x_{3}, \ldots\right\}$ be a countably infinite alphabet of variables and let $X_{n}=\left\{x_{1}, \ldots, x_{n}\right\}$ be an $n$-element alphabet. The $n$-ary terms of type $\tau$ are defined inductively as follows:

(i) the variables $x_{1}, \ldots, x_{n}$ are $n$-ary terms;

(ii) if $f_{i}$ is an $n_{i}$-ary operation symbol and $t_{1}, \ldots, t_{n_{i}}$ are $n$-ary terms, then $f_{i}\left(t_{1}, \ldots, t_{n_{i}}\right)$ is an $n$-ary term.

We will denote by $W_{\tau}\left(X_{n}\right)$ the set of $n$-ary terms of type $\tau$, that is, the smallest set which contains the variables $x_{1}, \ldots, x_{n}$ and which is closed under the finite application of (ii). It follows from this definition that any $n$-ary term is also $k$-ary for every $k \geq n$. The set $W_{\tau}(X)=\bigcup_{n=1}^{\infty} W_{\tau}\left(X_{n}\right)$ is the set of all terms of type $\tau$.

When the type $\tau$ is finitary, that is, when we have a finite number of operation symbols each of finite arity, we can represent each term of type $\tau$ by a tree diagram. Such trees have many applications in computer science, linguistics, and other fields. For such applications, it is important to measure the complexity of a term or a tree. The most commonly used measurement is that of the depth of a term (or dually, that of the height of a tree), and the method of algebraic induction often used in proofs about terms is based on this depth. But there are also several other natural complexity measures we can use.

In this paper, we examine the behaviour of various complexity measures under two mappings defined on sets of terms. The first such mapping is the 
operation of composition or superposition of terms, which plays an important role in universal algebra [5], clone theory [1, 7], and computer science [5, 6]. This is actually a family of operations: for each $m$ and $n$ in $\mathbb{N}$, the composition mapping $S_{m}^{n}$ maps one $n$-ary term and $n m$-ary terms onto an $m$-ary term as follows. We define

$$
S_{m}^{n}: W_{\tau}\left(X_{n}\right) \times W_{\tau}\left(X_{m}\right)^{n} \longrightarrow W_{\tau}\left(X_{m}\right)
$$

by the following steps, for $s \in W_{\tau}\left(X_{n}\right), t_{1}, \ldots, t_{n} \in W_{\tau}\left(X_{m}\right)$ and $f$, an operation symbol of arity $r$,

(i) if $s=x_{j}$ for $1 \leq j \leq n$, then $S_{m}^{n}\left(s, t_{1}, \ldots, t_{n}\right)=t_{j}$;

(ii) if $s=f\left(s_{1}, \ldots, s_{r}\right)$ and $s_{1}, \ldots, s_{r} \in W_{\tau}\left(X_{n}\right)$, then $S_{m}^{n}\left(s, t_{1}, \ldots, t_{n}\right)=$ $f\left(S_{m}^{n}\left(s_{1}, t_{1}, \ldots, t_{n}\right), \ldots, S_{m}^{n}\left(s_{r}, t_{1}, \ldots, t_{n}\right)\right)$.

The composition $S_{m}^{n}\left(s, t_{1}, \ldots, t_{n}\right)$ is often written as $s\left(t_{1}, \ldots, t_{n}\right)$. This operation is important in clone theory [1, 7], where the heterogeneous (multibased) algebra

$$
\left(W_{T}\left(X_{n}\right)_{n \in \mathbb{N}},\left(S_{m}^{n}\right)_{m, n \in \mathbb{N}},\left(x_{j}\right)_{j \leq n \in \mathbb{N}}\right),
$$

with the variables as nullary operations, is called the full-term clone of type $\tau$. (For technical reasons we usually exclude nullary terms, although it is possible to include them.)

We also consider mappings called hypersubstitutions on the set of all terms of a given type $\tau$. A hypersubstitution $\sigma$ of type $\tau$ is a mapping which assigns to each operation symbol $f_{i}$ of type $\tau$ an $n_{i}$-ary term $\sigma\left(f_{i}\right)$ of type $\tau$. Any such mapping $\sigma$ induces a unique mapping $\hat{\sigma}$ on the set of all terms of type $\tau$, given by the following inductive definition:

(i) $\hat{\sigma}[x]=x$ if $x \in X$ is a variable,

(ii) $\hat{\sigma}\left[f_{i}\left(t_{1}, \ldots, t_{n_{i}}\right)\right]=S_{m}^{n_{i}}\left(\sigma\left(f_{i}\right), \hat{\sigma}\left[t_{1}\right], \ldots, \hat{\sigma}\left[t_{n_{i}}\right]\right)$ for composite terms $f_{i}\left(t_{1}, \ldots, t_{n_{i}}\right)$.

The induced mapping $\hat{\sigma}$ is also often referred to as a hypersubstitution of type $\tau$ or as the extension of the hypersubstitution $\sigma$. Note that the second part of this definition uses the composition operation, making hypersubstitutions a special example of the use of the composition. We can define a binary operation ${ }^{\circ} h$ on the set $\operatorname{Hyp}(\tau)$ of all hypersubstitutions of type $\tau$ by letting $\sigma_{1}{ }^{\circ} \sigma_{2}$ be the hypersubstitution which maps each fundamental operation symbol $f_{i}$ to the term $\hat{\sigma}_{1}\left[\sigma_{2}\left(f_{i}\right)\right]$. The set $\operatorname{Hyp}(\tau)$ of all hypersubstitutions of type $\tau$ is closed under this associative binary operation. This set $\operatorname{Hyp}(\tau)$ is then a monoid with the identity hypersubstitution $\sigma_{\text {id }}$, which maps every $f_{i}$ to $f_{i}\left(x_{1}, \ldots, x_{n_{i}}\right)$, acting as an identity element. 
Now, let $M$ be any submonoid of $\operatorname{Hyp}(\tau)$. An identity $u \approx v$ of a variety $V$ is called an $M$-hyperidentity of $V$ if for every hypersubstitution $\sigma \in M$, the identity $\hat{\sigma}[u] \approx \hat{\sigma}[v]$ holds in $V$. A variety $V$ is called $M$-solid if every identity of $V$ is an $M$-hyperidentity of $V$. When $M$ is the whole monoid $\operatorname{Hyp}(\tau)$, an $M$ hyperidentity is called a hyperidentity and an $M$-solid variety is called a solid variety.

In the next section, we define four useful measurements of the complexity of a term. For each, we produce a formula for the complexity of the composition $S_{m}^{n}\left(s, t_{1}, \ldots, t_{n}\right)$ in terms of the complexity of the inputs $s, t_{1}, \ldots, t_{n}$. As a corollary, we also obtain formulas for the complexity of $\hat{\sigma}[t]$ in terms of the complexity of $t$ when $t$ is a compound term and $\sigma$ is a hypersubstitution. In the final section, we give an application of these formulas to the theory of $M$-solid varieties. We examine the chains obtained by taking the $k$-normalizations of a given variety $V$, as defined in [4], and show that under suitable choices of a monoid $N$, each variety of this chain is $(M \cap N)$-solid when the variety $V$ is $M$-solid. This can be used to produce infinite chains of $(M \cap N)$-solid varieties of any type.

2. Complexity of terms. To illustrate the various ways complexity of terms can be measured, we begin with an example. Throughout, we identify terms with the trees used to draw them.

EXAMPLE 2.1. Let $\tau$ be of type (3) with one ternary operation symbol $f$. Consider the term $t=f\left(x_{1}, f\left(x_{2}, x_{2}, x_{3}\right), f\left(f\left(x_{3}, x_{3}, x_{2}\right), x_{1}, x_{2}\right)\right)$. There are several numbers we can associate with $t$, each measuring a different aspect of how complex this term is as follows:

(i) the length of the longest path (from root to vertex) in $t$ is 3 ;

(ii) the length of the shortest path (from root to vertex) in $t$ is 1 ;

(iii) the total number of occurrences of variable symbols in $t$ is 9 ;

(iv) the number of distinct variables occurring in $t$ is 3 ;

(v) the number of occurrences of an operation symbol in $t$ is 4 .

Definition 2.2. (a) The maximum depth of a term $t$, which we denote by maxdepth $(t)$, is the length of the longest path from the root to a vertex in the tree. This is often called the depth of the tree. It is defined inductively by

(i) maxdepth $(t)=0$ if $t$ is a variable;

(ii) $\operatorname{maxdepth}(t)=1+\max \left\{\operatorname{maxdepth}\left(t_{j}\right): 1 \leq j \leq n_{i}\right\}$ if $t$ is a composite term $t=f_{i}\left(t_{1}, \ldots, t_{n_{i}}\right)$.

(b) The minimum depth of a term $t$, denoted by mindepth $(t)$, is the length of the shortest path from the root to a vertex in the tree and is defined inductively by

(i) mindepth $(t)=0$ if $t$ is a variable;

(ii) $\operatorname{mindepth}(t)=1+\min \left\{\operatorname{mindepth}\left(t_{j}\right): 1 \leq j \leq n_{i}\right\}$ if $t$ is a composite term $t=f_{i}\left(t_{1}, \ldots, t_{n_{i}}\right)$. 
(c) The variable count of a term $t$, denoted by $\mathrm{vb}(t)$, is the total number of occurrences of variables in $t$ (including multiplicities). This can be defined inductively by

(i) $\mathrm{vb}(t)=1$ if $t$ is a variable;

(ii) $\mathrm{vb}(t)=\sum_{j=1}^{n_{i}} \mathrm{vb}\left(t_{j}\right)$ if $t$ is a composite term $t=f_{i}\left(t_{1}, \ldots, t_{n_{i}}\right)$.

(d) The operation-symbol count of a term $t$, denoted by op $(t)$, is the total number of occurrences of operation symbols in $t$ and is inductively defined by

(i) $\mathrm{op}(t)=0$ if $t$ is a variable;

(ii) $\mathrm{op}(t)=1+\sum_{j=1}^{n_{i}} \mathrm{op}\left(t_{j}\right)$ if $t$ is a composite term $t=f_{i}\left(t_{1}, \ldots, t_{n_{i}}\right)$.

In all of these examples, we have a mapping $c: W_{\tau}(X) \rightarrow \mathbb{N}$ from the set of all terms of type $\tau$ to the set of natural numbers (including 0 ), which assigns to each term $t$ a complexity number $c(t)$. We refer to such a function as a complexity mapping or a cost function.

Before we can give our formulas for the complexity of a composed term, we need some subsidiary definitions and notation. Our complexity functions all measure the global complexity of a term, but we also need to consider how complex a term is with respect to a certain variable. That is, we also need to measure, for each variable $x_{j}$, both how many times it occurs in $t$ and the maximum and minimum depth at which it occurs. For any term $t \in W_{\tau}\left(X_{n}\right)$, let $\operatorname{var}(t)$ be the set of all variables occurring in the term $t$.

DEFINITION 2.3. Let $t \in W_{\tau}\left(X_{n}\right)$ be an $n$-ary term. For each variable $x_{k}$, we define the maximum depth $\operatorname{maxdepth}_{k}(t)$ with respect to $k$ of term $t$ inductively as follows:

(i) if $t$ is a variable from $X_{n}$, then $\operatorname{maxdepth}_{k}(t)=0$;

(ii) if $x_{k} \notin \operatorname{var}(t)$, then maxdepth $\operatorname{mon}_{k}(t)=0$;

(iii) if $t=f_{i}\left(t_{1}, \ldots, t_{n_{i}}\right)$ and $x_{k} \in \operatorname{var}(t)$, then $\operatorname{maxdepth}_{k}(t)=1+$ $\max \left\{\operatorname{maxdepth}_{k}\left(t_{j}\right) \mid 1 \leq j \leq n_{i}, x_{k} \in \operatorname{var}\left(t_{j}\right)\right\}$.

Analogously, we define the minimum depth with respect to $k$ for any term $t$ and any variable $x_{k}$.

DEFINITION 2.4. Let $t \in W_{\tau}\left(X_{n}\right)$ be an $n$-ary term. For each variable $x_{k}$, we define the minimum depth mindepth $_{k}(t)$ with respect to $k$ of term $t$ inductively as follows:

(i) if $t$ is a variable from $X_{n}$, then $\operatorname{mindepth}_{k}(t)=0$;

(ii) if $x_{k} \notin \operatorname{var}(t)$, then $\operatorname{mindepth}_{k}(t)=0$;

(iii) if $t=f_{i}\left(t_{1}, \ldots, t_{n_{i}}\right)$ and $x_{k} \in \operatorname{var}(t)$, then $\operatorname{mindepth}_{k}(t)=1+$ $\min \left\{\operatorname{mindepth}_{k}\left(t_{j}\right) \mid 1 \leq j \leq n_{i}, x_{k} \in \operatorname{var}\left(t_{j}\right)\right\}$.

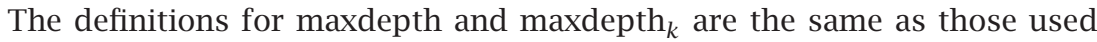
in [2], where they were referred to as depth formulas. It was also shown there that these mappings satisfy the equality

$$
\operatorname{maxdepth}(t)=\max \left\{\operatorname{maxdepth}_{k}(t) \mid 1 \leq k \leq n, x_{k} \in \operatorname{var}(t)\right\}
$$


for any $n$-ary term $t$. It is easy to verify that an analogous equality holds for mindepth and mindepth $_{k}$.

We also need a function that counts the number of occurrences of a specific variable $x_{k}$ in a term $t$.

DEFINITION 2.5. Let $t \in W_{\tau}\left(X_{n}\right)$ be an $n$-ary term. For each variable $x_{k}$, we define the $x_{k}$-variable count $\mathrm{vb}_{k}(t)$ of $t$ inductively as follows:

(i) $\operatorname{vb}_{k}\left(x_{k}\right)=1$;

(ii) if $x_{k}$ does not occur in $t$, then $\operatorname{vb}_{k}(t)=0$;

(iii) if $t=f_{i}\left(t_{1}, \ldots, t_{n_{i}}\right)$ and $x_{k} \in \operatorname{var}(t)$, then $\operatorname{vb}_{k}(t)=\sum_{j=1}^{n_{i}} \operatorname{vb}_{k}\left(t_{j}\right)$.

3. Complexity of composition and hypersubstitution. Now we are ready to give our complexity theorems. We remark that formula (b) for maxdepth was given by Denecke et al. in [2].

Proposition 3.1. Let $s \in W_{\tau}\left(X_{n}\right), t_{1}, \ldots, t_{n} \in W_{\tau}\left(X_{m}\right), 1 \leq n, m \in \mathbb{N}$. Then,

(a) $\operatorname{mindepth}\left(S_{m}^{n}\left(s, t_{1}, \ldots, t_{n}\right)\right)=\min \left\{\operatorname{mindepth}_{j}(s)+\operatorname{mindepth}\left(t_{j}\right) \mid 1 \leq j\right.$ $\left.\leq n, x_{j} \in \operatorname{var}(s)\right\}$

(b) $\operatorname{maxdepth}\left(S_{m}^{n}\left(s, t_{1}, \ldots, t_{n}\right)\right)=\max \left\{\operatorname{maxdepth}_{j}(s)+\operatorname{maxdepth}\left(t_{j}\right) \mid 1 \leq\right.$ $\left.j \leq n, x_{j} \in \operatorname{var}(s)\right\}$;

(c) $\operatorname{vb}\left(S_{m}^{n}\left(s, t_{1}, \ldots, t_{n}\right)\right)=\sum_{j=1}^{n} \mathrm{vb}_{j}(s) \mathrm{vb}\left(t_{j}\right)$;

(d) $\operatorname{op}\left(S_{m}^{n}\left(s, t_{1}, \ldots, t_{n}\right)\right)=\sum_{j=1}^{n} \operatorname{vb}_{j}(s)$ op $\left(t_{j}\right)+\operatorname{op}(s)$.

Proof. For convenience, we denote the term $S_{m}^{n}\left(s, t_{1}, \ldots, t_{n}\right)$ by $w$. We proceed throughout by induction on the structure of the term $s$, that is, on the maxdepth of $s$.

(a) If $s$ is a variable $x_{k}$ for some $1 \leq k \leq n$, then $S_{m}^{n}\left(s, t_{1}, \ldots, t_{n}\right)=t_{k}$ and thus $\operatorname{mindepth}\left(S_{m}^{n}\left(s, t_{1}, \ldots, t_{n}\right)\right)=\operatorname{mindepth}\left(t_{k}\right)$. Also we have $\operatorname{mindepth}_{j}(s)=0$ for all $1 \leq j \leq n$ and $x_{k}$ is the only variable to occur in $s$, so our formula gives

$\min \left\{\operatorname{mindepth}_{j}(s)+\operatorname{mindepth}\left(t_{j}\right) \mid 1 \leq j \leq n, x_{j} \in \operatorname{var}(s)\right\}=\operatorname{mindepth}\left(t_{k}\right)$.

Inductively, let $s=f\left(s_{1}, \ldots, s_{r}\right)$ and assume that the formula is satisfied for $s_{1}, \ldots, s_{r}$. Then, $w=S_{m}^{n}\left(s, t_{1}, \ldots, t_{n}\right)=f\left(S_{m}^{n}\left(s_{1}, t_{1}, \ldots, t_{n}\right), \ldots, S_{m}^{n}\left(s_{r}, t_{1}, \ldots, t_{n}\right)\right)$, and we have

$$
\begin{aligned}
& \operatorname{mindepth}\left(S_{m}^{n}\left(s, t_{1}, \ldots, t_{n}\right)\right) \\
& =\min \left\{\operatorname{mindepth}\left(S_{m}^{n}\left(s_{1}, t_{1}, \ldots, t_{n}\right)\right), \ldots, \operatorname{mindepth}\left(S_{m}^{n}\left(s_{r}, t_{1}, \ldots, t_{n}\right)\right)\right\}+1 \\
& =\min \left\{\operatorname { m i n } \left\{\operatorname{mindepth}_{j}\left(s_{1}\right)+\operatorname{mindepth}\left(t_{j}\right)\right.\right. \\
& \left.\mid 1 \leq j \leq n, x_{j} \in \operatorname{var}\left(s_{1}\right)\right\}, \ldots, \min \left\{\operatorname{mindepth}_{j}\left(s_{r}\right)\right. \\
& \left.\left.+\operatorname{mindepth}\left(t_{j}\right) \mid 1 \leq j \leq n, x_{j} \in \operatorname{var}\left(s_{r}\right)\right\}\right\}+1
\end{aligned}
$$




$$
\begin{aligned}
& =\min \left\{\min \left\{\operatorname{mindepth}_{j}\left(s_{k}\right) \mid 1 \leq k \leq r, x_{j} \in \operatorname{var}\left(s_{k}\right)\right\}\right. \\
& \left.\quad+1+\operatorname{mindepth}\left(t_{j}\right) \mid 1 \leq j \leq n, x_{j} \in \cup\left\{\operatorname{var}\left(s_{k}\right) \mid 1 \leq k \leq r\right\}\right\} \\
& =\min \left\{\min \left\{\operatorname{mindepth}_{j}\left(s_{k}\right) \mid 1 \leq k \leq r, x_{j} \in \operatorname{var}\left(s_{k}\right)\right\}\right. \\
& \quad+1+\operatorname{mindepth}_{\left.\left(t_{j}\right) \mid 1 \leq j \leq n, x_{j} \in \operatorname{var}(s)\right\}} \\
& =\min \left\{\operatorname{mindepth}_{j}(s)+\operatorname{mindepth}\left(t_{j}\right) \mid 1 \leq j \leq n, x_{j} \in \operatorname{var}(s)\right\} .
\end{aligned}
$$

(b) The proof in (a) for mindepth can be modified by replacing min by max throughout, to obtain a proof for maxdepth, as given in [2].

(c) If $s$ is a variable $x_{k}$ for some $1 \leq k \leq n$ then $S_{m}^{n}\left(s, t_{1}, \ldots, t_{n}\right)=t_{k}$ and thus $\operatorname{vb}\left(S_{m}^{n}\left(s, t_{1}, \ldots, t_{n}\right)\right)=\operatorname{vb}\left(t_{k}\right)$. Also we have $\operatorname{vb}_{j}(s)=0$ for all $1 \leq j \neq k \leq n$, so our formula gives

$$
\mathrm{vb}(w)=\sum_{j=1}^{n} \mathrm{vb}_{j}(s) \mathrm{vb}\left(t_{j}\right)=1 \cdot \mathrm{vb}\left(t_{k}\right)+0=\mathrm{vb}\left(t_{k}\right) .
$$

Inductively, let $s=f\left(s_{1}, \ldots, s_{r}\right)$ and assume that the formula is satisfied for $s_{1}, \ldots, s_{r}$. Then,

$$
\begin{aligned}
\operatorname{vb}(w) & =\operatorname{vb}\left(S_{m}^{n}\left(s, t_{1}, \ldots, t_{n}\right)\right) \\
& =\operatorname{vb}\left(f\left(S_{m}^{n}\left(s_{1}, t_{1}, \ldots, t_{n}\right), \ldots, S_{m}^{n}\left(s_{r}, t_{1}, \ldots, t_{n}\right)\right)\right) \\
& =\sum_{k=1}^{r} \operatorname{vb}\left(S_{m}^{n}\left(s_{k}, t_{1}, \ldots, t_{n}\right)\right) \\
& =\sum_{k=1}^{r}\left(\sum_{j=1}^{n} \operatorname{vb}_{j}\left(s_{k}\right) \operatorname{vb}\left(t_{j}\right)\right) \\
& =\sum_{j=1}^{n}\left(\sum_{k=1}^{r} \operatorname{vb}_{j}\left(s_{k}\right) \operatorname{vb}\left(t_{j}\right)\right) \\
& =\sum_{j=1}^{n}\left(\left(\sum_{k=1}^{r} \operatorname{vb}_{j}\left(s_{k}\right)\right) \operatorname{vb}\left(t_{j}\right)\right) \\
& =\sum_{j=1}^{n} \operatorname{vb}_{j}(s) \mathrm{vb}\left(t_{j}\right) .
\end{aligned}
$$

(d) If $s$ is a variable $x_{k}$ for some $1 \leq k \leq n$, then op $\left(S_{m}^{n}\left(s, t_{1}, \ldots, t_{n}\right)\right)=\operatorname{op}\left(t_{k}\right)$. Also we have $\operatorname{op}(s)=0$ and $\operatorname{vb}_{j}(s)=0$ for all $1 \leq j \neq k \leq n$, so our formula gives

$$
\mathrm{op}(w)=\sum_{j=1}^{n} \mathrm{vb}_{j}(s) \mathrm{op}\left(t_{j}\right)+\mathrm{op}(s)=1 \cdot \mathrm{op}\left(t_{k}\right)+0=\mathrm{op}\left(t_{k}\right)
$$


Inductively, let $s=f\left(s_{1}, \ldots, s_{r}\right)$ and assume that the formula is satisfied for $s_{1}, \ldots, s_{r}$. Then,

$$
\begin{aligned}
\operatorname{op}(w) & =\operatorname{op}\left(S_{m}^{n}\left(s, t_{1}, \ldots, t_{n}\right)\right) \\
& =\operatorname{op}\left(f\left(S_{m}^{n}\left(s_{1}, t_{1}, \ldots, t_{n}\right), \ldots, S_{m}^{n}\left(s_{r}, t_{1}, \ldots, t_{n}\right)\right)\right) \\
& =1+\sum_{k=1}^{r} \mathrm{op}\left(S_{m}^{n}\left(s_{k}, t_{1}, \ldots, t_{n}\right)\right) \\
& =1+\sum_{k=1}^{r}\left(\left(\sum_{j=1}^{n} \mathrm{vb}_{j}\left(s_{k}\right) \mathrm{op}\left(t_{j}\right)\right)+\mathrm{op}\left(s_{k}\right)\right) \\
& =1+\sum_{k=1}^{r} \mathrm{op}\left(s_{k}\right)+\sum_{k=1}^{r}\left(\sum_{j=1}^{n} \mathrm{vb}_{j}\left(s_{k}\right) \mathrm{op}\left(t_{j}\right)\right) \\
& =\operatorname{op}(s)+\sum_{j=1}^{n}\left(\sum_{k=1}^{r} \mathrm{vb}_{j}\left(s_{k}\right) \mathrm{op}\left(t_{j}\right)\right) \\
& =\operatorname{op}(s)+\sum_{j=1}^{n}\left(\left(\sum_{k=1}^{r} \mathrm{vb}_{j}\left(s_{k}\right)\right) \mathrm{op}\left(t_{j}\right)\right) \\
& =\sum_{j=1}^{n} \operatorname{vb}_{j}(s) \mathrm{op}\left(t_{j}\right)+\mathrm{op}(s) .
\end{aligned}
$$

Using the fact that the hypersubstitution $\hat{\sigma}[t]$ is defined using composition, we have the following corollary.

COROLLARY 3.2. Let $t$ be a composite term of the form $t=f\left(t_{1}, \ldots, t_{n}\right)$, where $f$ is an n-ary operation symbol. Let $\sigma$ be a hypersubstitution of type T. Then,

(a) $\operatorname{maxdepth}(\hat{\sigma}[t])=\max \left\{\operatorname{maxdepth}_{j}(\hat{\sigma}(f))+\operatorname{maxdepth}\left(\hat{\sigma}\left[t_{j}\right]\right) \mid 1 \leq j \leq\right.$ $\left.n, x_{j} \in \operatorname{var}(\sigma(f))\right\}$;

(b) $\operatorname{mindepth}(\hat{\sigma}[t])=\min \left\{\operatorname{mindepth}_{j}(\hat{\sigma}(f))+\operatorname{mindepth}\left(\hat{\sigma}\left[t_{j}\right]\right) \mid 1 \leq j \leq\right.$ $\left.n, x_{j} \in \operatorname{var}(\sigma(f))\right\}$

(c) $\operatorname{vb}(\hat{\sigma}[t])=\sum_{j=1}^{n} \operatorname{vb}_{j}(\sigma(f)) \operatorname{vb}\left(\hat{\sigma}\left[t_{j}\right]\right)$;

(d) $\operatorname{op}(\hat{\sigma}[t])=\sum_{j=1}^{n} \operatorname{vb}_{j}(\sigma(f)) \operatorname{op}\left(\hat{\sigma}\left[t_{j}\right]\right)+\operatorname{op}(\sigma(f))$.

4. $M$-Solid varieties. In this section, we give an application of our formulas for complexity of compositions and hypersubstitutions to the study of $M$-solid varieties. We consider the so-called $k$-normalizations $N_{k}(V)$ of a given variety $V$, defined by Denecke et al. [3] and Denecke and Wismath [4]. In particular, we describe the $M$-solidity of these varieties in terms of the $M$-solidity of $V$.

We begin with some notation needed to discuss the $k$-normalization of a variety. For any variety $V$ of a fixed type $\tau$, we denote by $\operatorname{Id} V$ the set of all identities of type $\tau$ satisfied by $V$, and for any set $\Sigma$ of identities of type $\tau$, we denote by $\operatorname{Mod} \Sigma$ the variety of all algebras of type $\tau$ which satisfy all the identities in $\Sigma$. Now let $V$ be a variety of type $\tau$ and let $k$ be a natural number, 
$k \geq 0$. Let $c$ be one of the four complexity functions defined in Section 2 . We define the $k$-normalization of $V$, with respect to the complexity function $c$, to be the variety $N_{k}^{c}(V)=\operatorname{Mod}\{u \approx v \in \operatorname{Id} V \mid c(u), c(v) \geq k\}$.

It is clear that $N_{0}^{c}(V)=V$ and that the $k$-normalizations of $V$ form a chain

$$
V=N_{0}^{c}(V) \leq N_{1}^{c}(V) \leq N_{2}^{c}(V) \leq \cdots
$$

The properties of these varieties, and of the operator $N_{k}^{c}$ for $k \geq 0$, have been studied for $c=$ mindepth in [3] and for $c=$ maxdepth in [4].

Our goal now is to examine the $M$-solidity properties of the varieties $N_{k}^{c}(V)$. Suppose that we start with an $M$-solid variety $V$ of type $\tau$ for some monoid $M$ of hypersubstitutions of type $\tau$. What can be said about the $M$-solidity of the variety $N_{k}^{c}(V)$ for $k \geq 1$ ? To show that $N_{k}^{c}(V)$ is also $M$-solid, we have to show that for any identity $u \approx v$ of $N_{k}^{c}(V)$ and any $\sigma \in M$, we have $\hat{\sigma}[u] \approx \hat{\sigma}[v]$ also in $\operatorname{Id} N_{k}^{c}(V)$. It suffices to consider an identity $u \approx v$ from the defining basis for $N_{k}^{c}(V)$, that is, we may assume that $u \approx v$ is an identity of $V$ with the property that both $c(u)$ and $c(v)$ are greater than or equal to $k$. Since $V$ itself is $M$-solid, we know that $\hat{\sigma}[u] \approx \hat{\sigma}[v]$ is in $\operatorname{Id} V$. Thus, it suffices to show that both $c(\hat{\sigma}[u])$ and $c(\hat{\sigma}[v])$ are also greater than or equal to $k$. In general, then, we need to compare the complexity of a term $t$ with the complexity of $\hat{\sigma}[t]$ and would like to be able to show that $c(\hat{\sigma}[t]) \geq c(t)$. However, this is not always the case as the following example shows.

EXAMPLE 4.1. (a) Consider the type $\tau=(2)$ with one binary operation symbol $f$. Let $t$ be the term $f(x, f(y, z))$ so that maxdepth $(t)=2$, mindepth $(t)=$ $1, \operatorname{vb}(t)=3$, and op $(t)=2$. Let $\sigma$ be the hypersubstitution mapping $f$ onto the term $f\left(x_{1}, x_{1}\right)$. Then, we have $\hat{\sigma}[t]=f(x, x)$, and this term has maxdepth $=$ mindepth $=$ op $=1$ and $\mathrm{vb}=2$. Thus, all but mindepth result in lower complexity for $\hat{\sigma}[t]$ than for $t$.

(b) Now let $\tau=(2,2)$ with two binary symbols $f$ and $g$. Let $t=f(f(x, y)$, $g(x, y)$ ), and let $\sigma$ be the hypersubstitution which maps $f$ onto the term $f\left(x_{2}, x_{2}\right)$ and $g$ onto the variable $x_{1}$. Then, although $t$ has mindepth $=2$, the term $\hat{\sigma}[t]=f(x, x)$ has mindepth of 1 .

Although not all hypersubstitutions $\sigma$ have the property that $\hat{\sigma}[t]$ has a complexity greater than or equal to the complexity of $t$, there are conditions we can put on $\sigma$ to ensure this property. For our complexity functions, two properties of hypersubstitutions turn out to be important, namely, regularity and pre-hypersubstitutions. A hypersubstitution $\sigma \in \operatorname{Hyp}(\tau)$ is called regular if for every $i \in I$, all the variables $x_{1}, \ldots, x_{n_{i}}$ occur in the term $\sigma\left(f_{i}\right)$. The set $\operatorname{Reg}(\tau)$ of all regular hypersubstitutions of type $\tau$ forms a submonoid of $\operatorname{Hyp}(\tau)$, and a variety which is $M$-solid for this submonoid $M$ is called regular-solid. A prehypersubstitution of type $\tau$ is a hypersubstitution $\sigma$ with the property that for every operation symbol $f_{i}$ of the type, $\sigma\left(f_{i}\right)$ is not a variable. The set $\operatorname{Pre}(\tau)$ 
of all pre-hypersubstitutions of type $\tau$ forms a submonoid of $\operatorname{Hyp}(\tau)$, and a variety which is $M$-solid for this monoid is said to be presolid.

THEOREM 4.2. Let $t \in W_{\tau}(X)$ be a term and $\sigma$ be a hypersubstitution of type T. Then,

(a) if $\sigma$ is regular, then maxdepth $(\hat{\sigma}[t]) \geq \operatorname{maxdepth}(t)$;

(b) if $\sigma$ is regular, then $\mathrm{vb}(\hat{\sigma}[t]) \geq \mathrm{vb}(t)$;

(c) if $\sigma$ is regular, then op $(\hat{\sigma}[t]) \geq \mathrm{op}(t)$;

(d) if $\sigma$ is a pre-hypersubstitution, then mindepth $(\hat{\sigma}[t]) \geq \operatorname{mindepth}(t)$.

Proof. We prove all of the four claims by induction on the structure of the term $t$. In all cases, when $t$ is a variable $x$, we have $\hat{\sigma}[t]=x=t$, and both $\hat{\sigma}[t]$ and $t$ have the same complexity.

Inductively, let $t=f_{i}\left(t_{1}, \ldots, t_{n_{i}}\right)$ for some $n_{i}$-ary operation symbol $f_{i}$ of type $\tau$ so that $\hat{\sigma}[t]=\sigma(f)\left(\hat{\sigma}\left[t_{1}\right], \ldots, \hat{\sigma}\left[t_{n}\right]\right)$. Now we apply the formulas from Corollary 3.2 to this. Note that when $\sigma$ is regular, we have $x_{j} \in \operatorname{var}\left(\sigma\left(f_{i}\right)\right)$, maxdepth $_{j}\left(\sigma\left(f_{i}\right)\right) \geq 1$, and $\operatorname{vb}_{j}\left(\sigma\left(f_{i}\right)\right) \geq 1$ for all $1 \leq j \leq n_{i}$, and also have op $\left(\sigma\left(f_{i}\right)\right) \geq 1$.

(a) For $c=$ maxdepth we have

$$
\begin{aligned}
\operatorname{maxdepth}(\hat{\sigma}[t])= & \max \left\{\operatorname{maxdepth}_{j}\left(\sigma\left(f_{i}\right)\right)+\operatorname{maxdepth}\left(\hat{\sigma}\left[t_{j}\right]\right) \mid 1 \leq j \leq n_{i},\right. \\
& \left.x_{j} \in \operatorname{var}\left(\sigma\left(f_{i}\right)\right)\right\} \\
= & \max \left\{\operatorname{maxdepth}_{j}\left(\sigma\left(f_{i}\right)\right)+\operatorname{maxdepth}\left(\hat{\sigma}\left[t_{j}\right]\right) \mid 1 \leq j \leq n_{i}\right\} \\
\geq & \text { since } \sigma \text { is regular } \\
\geq & 1+\max \left\{\operatorname{maxdepth}\left(\hat{\sigma}\left[t_{j}\right]\right) \mid 1 \leq j \leq n_{i}\right\} \quad \text { since } \sigma \text { is regular } \\
= & \operatorname{maxdepth}(t) .
\end{aligned}
$$

(b) For $c=$ vb we have

$$
\begin{aligned}
\operatorname{vb}(\hat{\sigma}[t]) & =\sum_{j=1}^{n_{i}} \operatorname{vb}_{j}\left(\sigma\left(f_{i}\right)\right) \mathrm{vb}\left(\hat{\sigma}\left[t_{j}\right]\right) \\
& \geq \sum_{j=1}^{n_{i}} 1 \cdot \operatorname{vb}\left(t_{j}\right) \quad \text { by induction and regularity } \\
& =\sum_{j=1}^{n_{i}} \mathrm{vb}\left(t_{j}\right)=\mathrm{vb}(t) .
\end{aligned}
$$


(c) For $c=$ op we have

$$
\begin{aligned}
\mathrm{op}(\hat{\sigma}[t]) & =\sum_{j=1}^{n_{i}} \operatorname{vb}_{j}\left(\sigma\left(f_{i}\right)\right) \mathrm{op}\left(\hat{\sigma}\left[t_{j}\right]\right)+\mathrm{op}\left(\sigma\left(f_{i}\right)\right) \\
& \geq \sum_{j=1}^{n_{i}} 1 \cdot \mathrm{op}\left(t_{j}\right)+1 \quad \text { by induction and regularity } \\
& =\sum_{j=1}^{n_{i}} \mathrm{op}\left(t_{j}\right)+1=\mathrm{op}(t) .
\end{aligned}
$$

(d) Note that when $\sigma\left(f_{i}\right)$ is not a variable, we must have mindepth m $_{j}\left(\sigma\left(f_{i}\right)\right) \geq$ 1 for all indices $j$ such that $x_{j} \in \operatorname{var}\left(\sigma\left(f_{i}\right)\right)$. Then, we have

$$
\begin{aligned}
\operatorname{mindepth}(\hat{\sigma}[t])= & \min \left\{\operatorname{mindepth}_{j}\left(\sigma\left(f_{i}\right)\right)+\operatorname{mindepth}\left(\hat{\sigma}\left[t_{j}\right]\right) \mid 1 \leq j \leq n_{i},\right. \\
& \left.x_{j} \in \operatorname{var}\left(\sigma\left(f_{i}\right)\right)\right\} \\
\geq & 1+\min \left\{\operatorname{mindepth}\left(\hat{\sigma}\left[t_{j}\right]\right) \mid 1 \leq j \leq n_{i}, x_{j} \in \operatorname{var}\left(\sigma\left(f_{i}\right)\right)\right\} \\
\geq & 1+\min \left\{\operatorname{mindepth}\left(t_{j}\right) \mid 1 \leq j \leq n_{i},\right. \\
& \left.x_{j} \in \operatorname{var}\left(\sigma\left(f_{i}\right)\right)\right\} \quad \text { by induction } \\
\geq & 1+\min \left\{\operatorname{mindepth}\left(t_{j}\right) \mid 1 \leq j \leq n_{i}\right\} \\
= & \operatorname{mindepth}(t) .
\end{aligned}
$$

Combining this theorem with the discussion preceding Theorem 4.2 gives the following result.

COROLLARY 4.3. Let $V$ be a nontrivial $M$-solid variety of type $\tau$. Then for all $k \geq 1$,

(a) for $c=$ maxdepth, each $N_{k}^{c}(V)$ is ( $\left.M \cap \operatorname{Reg}\right)$-solid;

(b) for $c=v b$, each $N_{k}^{c}(V)$ is $(M \cap \operatorname{Reg})$-solid;

(c) for $c=$ op, each $N_{k}^{c}(V)$ is $(M \cap \operatorname{Reg})$-solid;

(d) for $c=$ mindepth, each $N_{k}^{c}(V)$ is $(M \cap$ Pre) -solid;

(e) for $c=$ mindepth if $\tau=(n)$ for some $n \geq 2$, then each $N_{k}^{c}(V)$ is $M$-solid.

A special case of part (e) of this corollary, taking $M$ to be the monoid $\operatorname{Hyp}(n)$ of all hypersubstitutions of type $(n)$, was used in [3] to show that there are infinitely many solid varieties of type $(n)$. Many of the examples given there, of varieties $V$ for which the chains $N_{k}^{c}(V)$ are infinite when $c=$ mindepth, may be modified to serve the other choices of $c$ as well.

We remark that in [8] a somewhat similar process has been considered using the arity of a term as a complexity function, but using descending, rather than ascending, chains of varieties. The varieties studied there were of the form

$$
A_{k}^{\mathrm{ar}}(V)=\operatorname{Mod}\{u \approx v \in \operatorname{Id} V \mid u, v \text { both have arity }=k\} .
$$


Since any term of an arity $k$ is also $n$-ary for any $n \geq k$, this gives the descending chain of varieties $A_{k}^{\mathrm{ar}}(V) \geq A_{k+1}^{\mathrm{ar}}(V)$, all containing $V$. The solidity of these varieties was related to another type of solidity, called $n$-solidity of a variety.

Acknowledgment. This research was supported by the Natural Sciences and Engineering Research Council (NSERC) of Canada.

\section{REFERENCES}

[1] G. Birkhoff and J. D. Lipson, Heterogeneous algebras, J. Combinatorial Theory 8 (1970), 115-133.

[2] K. Denecke, J. Koppitz, and S. Shtrakov, The depth of a hypersubstitution, J. Autom. Lang. Comb. 6 (2001), no. 3, 253-262.

[3] K. Denecke, J. Koppitz, and S. L. Wismath, Solid varieties of arbitrary type, to appear in Algebra Universalis.

[4] K. Denecke and S. L. Wismath, Complexity of Terms and the Galois Connection Id-Mod, preprint, 2001.

[5] Universal Algebra and Applications in Theoretical Computer Science, Chapman \& Hall, Florida, 2002.

[6] F. Gécseg and M. Steinby, Tree Automata, Akadémiai Kiadó, Budapest, 1984.

[7] P. J. Higgins, Algebras with a scheme of operators, Math. Nachr. 27 (1963), 115132.

[8] S. L. Wismath, Fundamental-M-solid and fundamental-M-closed varieties, Southeast Asian Bull. Math. 25 (2001), no. 1, 161-174.

Klaus Denecke: Institut für Mathematik, Universität Potsdam, Am Neuen Palais, 14415 Potsdam, Germany

E-mail address: kdenecke@rz.uni-potsdam.de

Shelly L. Wismath: Department of Mathematics and Computer Science, University of Lethbridge, Lethbridge, Alberta, Canada T1K 3M4

E-mail address: wi smaths@cs.u1eth.ca 


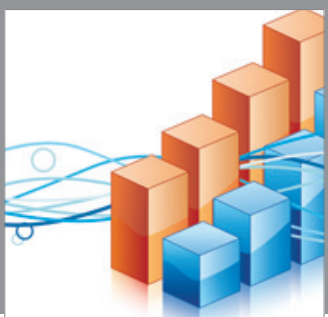

Advances in

Operations Research

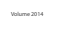

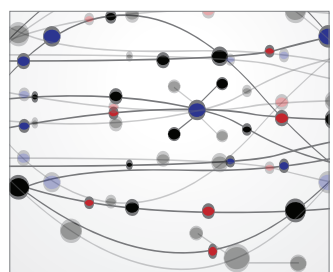

\section{The Scientific} World Journal
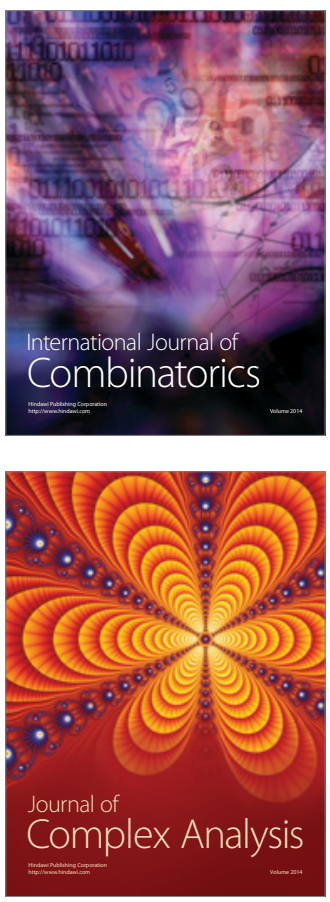

International Journal of

Mathematics and

Mathematical

Sciences
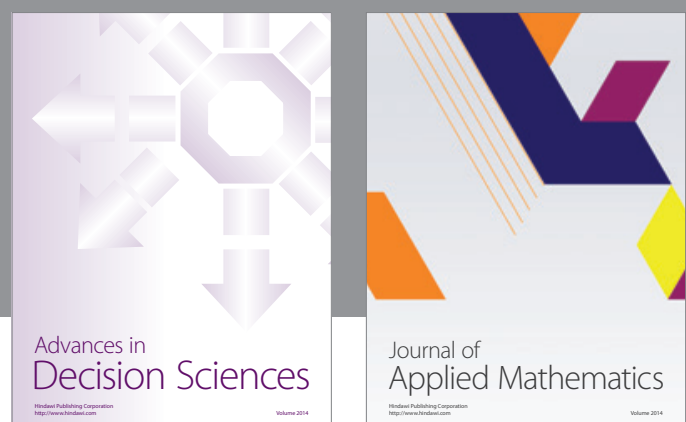

Journal of

Applied Mathematics
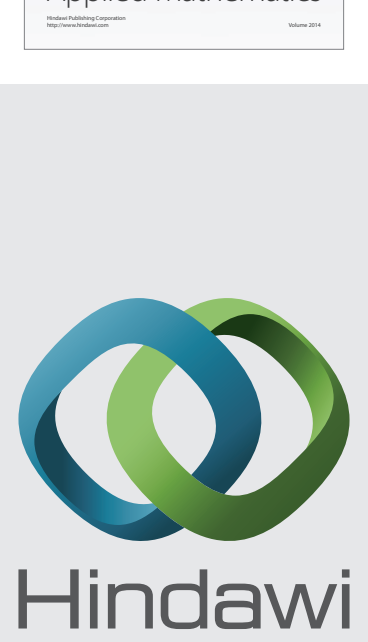

Submit your manuscripts at http://www.hindawi.com
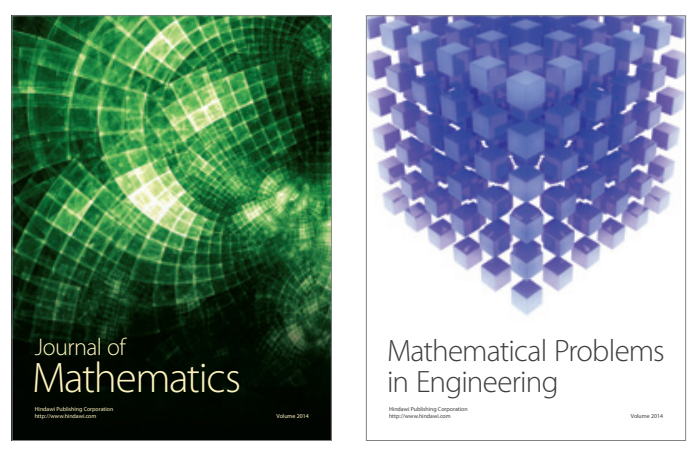

Mathematical Problems in Engineering
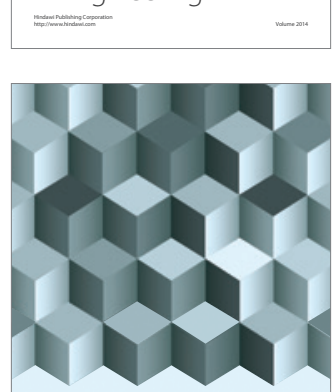

Journal of

Function Spaces
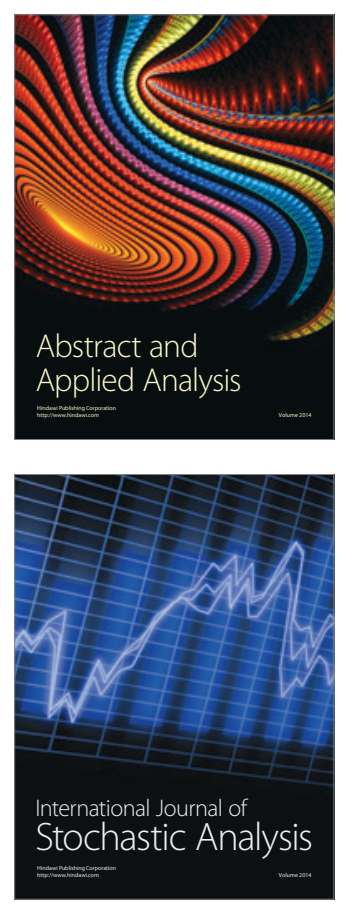

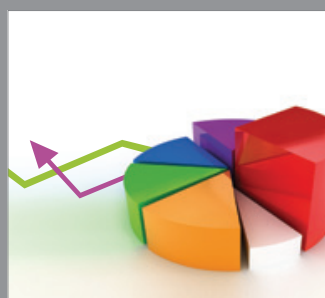

ournal of

Probability and Statistics

Promensencen
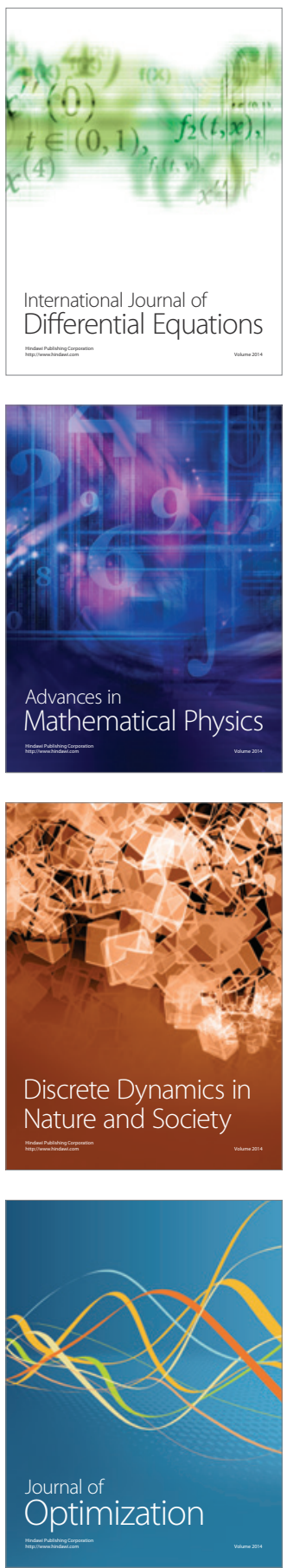